\title{
Fixation-Based Surgery: A New Technique for Distal Radius Osteotomy
}

\author{
H. Croitoru \\ R.E. Ellis \\ C.F. Small \\ D.R. Pichora \\ Computing and Information Science \\ Mechanical Engineering \\ Surgery \\ Queen's University at Kingston, Canada \\ ellis@cs.queensu.ca
}

\begin{abstract}
Fixation-based surgery is a new technique for achieving difficult corrections in some orthopedic procedures. The method is premised on using a fixation device, such as a fracture-fixation plate, during the alignment and distration phases of an open-wedge osteotomy. The basic idea is similar to assembly of manufactured components: pilot holes are drilled in the bone fragments, the fixation plate is attached to one fragment and, when the fragment is moved, the alignment has been achieved when the pilot holes in one fragment line up with predetermined through holes in the fixation plate.

The method has been specifically developed to address osteotomy of the distal radius to correct a malunited fracture. The method has been validated in laboratory studies. Clinical trials suggest that the method is no slower than the conventional technique, there is almost no intraoperative X-ray exposure, and that exceptionally large corrections can easily be achieved.
\end{abstract}

\section{Rationale and Objectives}

Fractures of the distal radius constitute about $15 \%$ of all fractures seen in the emergency room $[4,6]$. The distal bone fragment may fail to realign to its proper anatomical position during healing, which can affect the alignment, kinematics, and load transfer across the wrist. Such malunions often lead to reduced strength, reduced range of motion, and pain. Correction of a malunion is performed using distal radius osteotomy (DRO). This procedure involves cutting the distal radius near its original fracture site and realigning it to restore normal function. For optimal outcome, proper planning is important to ensure that all the proper lengths and angles are restored. We use a patient-specific measure, in which the affected wrist is realigned to match the healthy wrist.

\subsection{Traditional Technique}

In traditional technique, AP and lateral X-ray films of the deformed wrist are taken. Osteotomy lines are drawn on the X-ray films to determine the size and shape of the bone graft [1] needed to correct the wrist to accepted radiographic indices. Intraoperatively, after the bone has been cut, fluoroscopic images are taken to determine the alignment of the distal bone fragment. This process is repeated until the desired orientation and position is obtained. The defect gap is filled with a bone graft or bone substitute. A fixation plate is contoured to the shape of the distal and proximal fragments, secured with bone screws. 


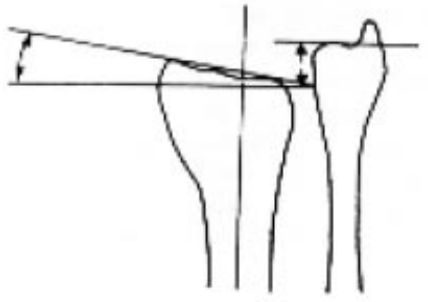

A

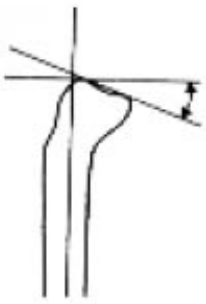

B

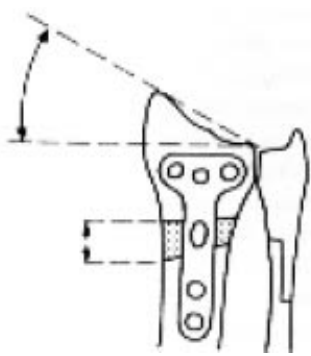

C

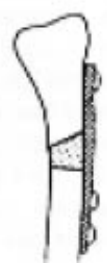

D

Fig. 1. Distal Radius Osteotomy. (A) A malunion typically exhibits shortening and an radial tilt of the radius. (B) Dorsal tilt may also be exhibited. (C,D) After correction, a wedge of bone fills the gap that is created by extending and realigning the distal radius.

This technique has three major limitations: poor planning of rotations in three dimensions; difficulty in achieving the desired lengthening, due to soft-tissue contracture; and a high dosage of fluoroscopic radiation to the patient and OR staff during the surgery.

\subsection{Previous Computer-Assisted Techniques}

To date, two studies have attempted to overcome these limitations. The BIZCAD system created by Bilić and Zdravković [2, 3, 12] used data obtained from two perpendicular $\mathrm{X}$-ray films to create 3D wireframe models of the healthy and deformed radii. These models were constructed from fourteen landmarks that were marked on the X-ray films and later digitized. Despite positive outcome of seven trial cases this system limited: there were potential data-entry errors in entering the coordinates of the landmark locations, and the system was only a planning system (there was no way to inform the surgeon during the operation as to where the exact location and orientation of the osteotomy should be).

Jupiter et al. [9] used CAD/CAM technology and CT data to generate plastic models of the wrist to visualize and complex malunions. This approach was useful in envisioning corrections of complex malunions, but offered little help in determining the quantitative data necessary to plan the correction. Use of this method was also limited due to the high cost of creating the models.

\subsection{A Fixation-Based Computer-Assisted Technique}

We have developed a technique that is a fundamental departure from all previously reported techniques. By analysing the geometry of the completed osteotomy, we have determined that there are three components to a successful precedure: 
Distraction of the fragment, which requires stretching soft tissues that have contracted; Alignment of the fragment to restore the distal radius to its correct anatomical location; and

Fixation of the fragment to preserve the alignment.

Our computer-assisted system requires a preoperative plan of the alignment and fixation of an uncontoured plate. Intraoperatively, pilot holes are drilled into the bone and the bone is shaved to fit the plate. When the through holes in the plate align with the pilot holes in the bone, the correct alignment has been achieved and the plate is in the correct position for fixation. The defect is subsequently filled with bone graft or bone substitute.

This is a radical departure from the traditional technique. Fixation-based surgery requires computer assistance in both planning and guidance, which requires additional preoperative time for the planning process. However, the operative time is no greater than that needed for the traditional technique, there is almost no use of intraoperative X-ray fluoroscopy, and exceptionally large defects can be corrected. We have conducted an in vitro study that showed significant improvements over the traditional method. We have also performed four clinical cases that demonstrate the practicality of our method.

\section{Materials and Methods}

Five steps are involved in our procedure. Preoperatively, the patient was scanned, models were generated, and the surgical plan was formulated. Intraoperatively, the patient's anatomy was registered to the preoperative data and image-guided surgery was performed.

1. Patient Scanning: Both patient wrists were scanned in neutral rotation using helical CT. Scans were also acquired from the proximal radii and ulnae to help in the long bone alignment.

2. 3D Model Generation: Voxel intensities were extracted from the scan and used to create isosurface models [5]. The models of the healthy radius and ulna were reflected to serve as a template for the correction.

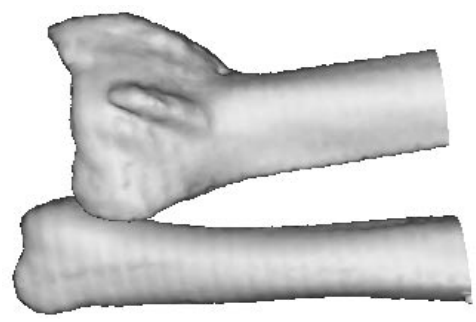

The ulna and the malunited radius 


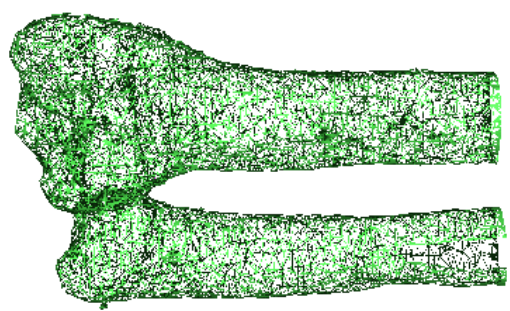

Mirror image of the healthy arm acts as a template

3. Plan Creation: The planning system used an X-Windows interface and the OpenGL 1.11 graphics library. Creating a preoperative plan involved five steps:

(a) Initial Alignment The entire ulna and the proximal radius of the affected wrist were aligned with the template. The affected radius demonstrated a deformity.

(b) Virtual Osteotomy A cutting plane was chosen by the surgeon and the isosurface model was cut into two models.

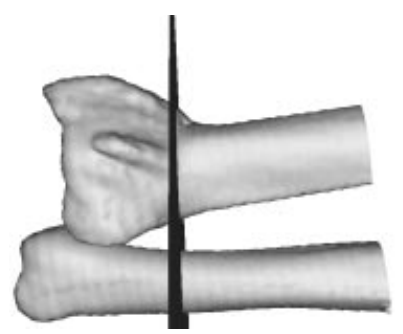

(c) Aligning the Distal Radii The distal radial fragment of the deformed radius was aligned with the healthy distal radius. Of particular interest were the subchondral arc that was formed between the ulna and radius and the ulnar variance.

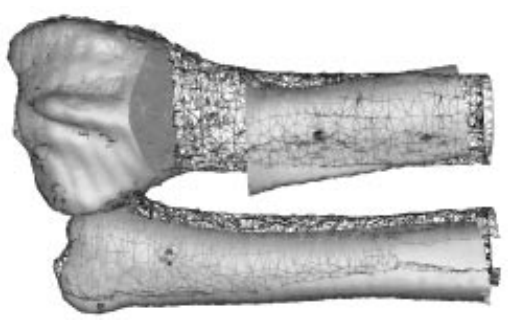

(d) Positioning the Fixation Plate Once the surgeon was satisfied with the new alignment of the deformed distal radius, the model of the fixation plate was placed on the models such that in vivo it would hold the bone fragments in place. In placing the fixation plate on the bones, it was important to verify that it lay flat on the bones and that none of the screw holes for the plate would protrude into any joints. 


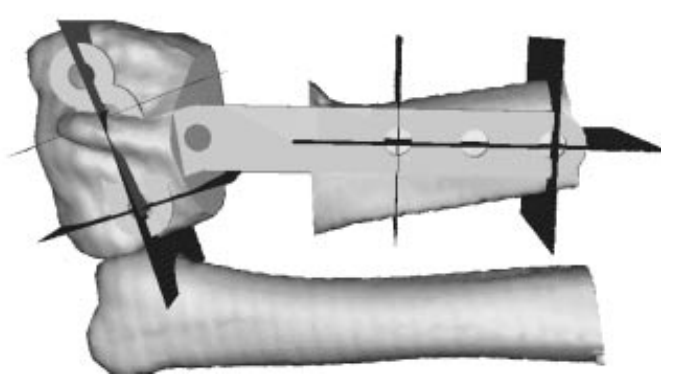

(e) Saving the Plan The location of the two distal and proximal drill holes for the plate were saved. The locations of the distal holes before the cutting of the bone were determined by applying the inverse transformation of the distal fragment to the drill locations.

4. Intraoperative Registration: We used a robust registration method to estimate the rigid-body transformation from the anatomy to the preoperative data [10]. While the patient was being anesthetized, the surgeon selected initial-estimate landmarks ("spotlights"). Intraoperatively the surgeon contacted the bone in these spotlights, which provided the registration algorithm with a good initial estimate of the registration. The surgeon then collected about 10 additional surface points to refine the registration estimate. A sequence of robust estimators were used to discard statistical outliers from the data in a mathematically disciplined manner.

5. Image-Guided Surgery: The 3D bone and plate models, CT data, and preoperative plan are imported into the guidance system. An OPTOTRAK 3020 (Northern Digital Inc., Waterloo, Canada) was used for tracking the surgical tools and patient movements. The sequence of the surgical procedure was:

- Drill proximal and distal pilot holes for the fixation plate with image guidance, with the locations of the pilot holes dictated by the preoperatively planned location of the plate;

- Cut the bone (with image guidance);

- Affix the plate to the distal radius fragment;

- Implant a temporary anchor screw into the radial midshaft, proximal to the plate;

- Progressively distract the plate by "jacking" the plate against the anchor screw with a laminar spreader;

- Align the screw holes in the plate with the pilot holes in the bone;

- Secure the plate to the proximal fragment and remove the anchor screw; and

- Fill the defect with autologous bone graft. 


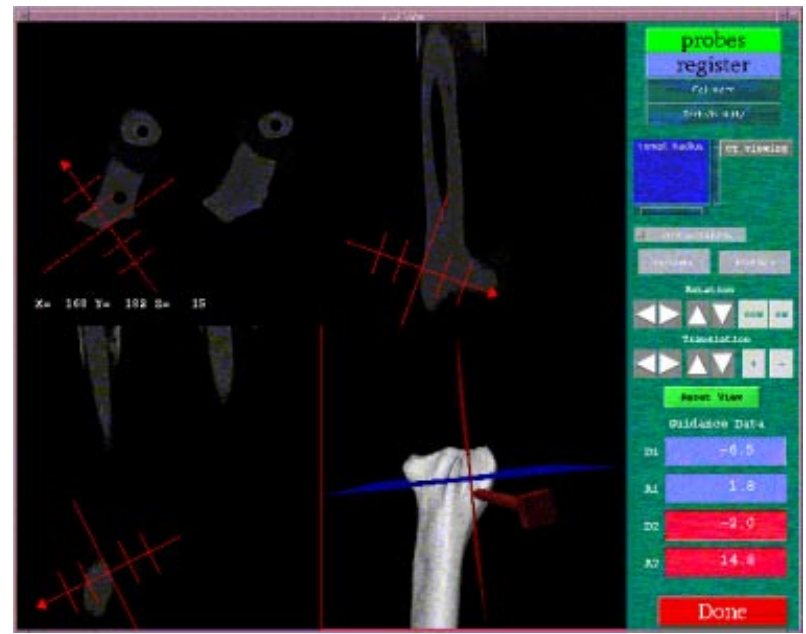

Image guidance for drilling a pilot hole for the fixation plate

\subsection{A Laboratory Study}

Fourteen identical polyurethane-foam models of a single deformed radius (model\#1029, Pacific Research Laboratories, Bellingham, WA), one normal radius (model\#1018), and two normal ulnae (model \#1017) were used for the study. One normal ulna and radii were affixed in neutral rotation and scanned to represent the template for correction. A jig was constructed for the deformed radii to ensure that they would always lie in the exact location relative to the ulna.

Seven corrections were performed using the traditional procedure using fluoroscopy and seven using the computer planning and navigation system. Corrective procedures were alternated to reduce potential bias in the results.

In each of the procedures, a fresh deformed radius was fastened in the jig and infrared emitting diode (IRED) markers were attached to the distal radial bone fragment and the radial shaft. The locations of the two markers were captured before and after the procedure and used to analyze the overall correction established. The data collected from the marker locations were analyzed for the translational and rotational corrections. Mean, Standard Deviation, and range were computed for each spatial degree of freedom.

\section{Results}

Statistical analyses were performed using the Statistical Package for the Social Sciences (SPSS Inc., Chicago, IL). For accuracy, the mean translations and rotations were analyzed using Student's t-test; in cases where there was a significant difference in the variance between the computer-assisted and traditional group results, a Kruskal-Wallis $\mathrm{H}$ test for two samples (equivalent to the Mann-Whitney $\mathrm{U}$ test) was used to analyze the accuracy. For repeatability, standard deviations were analyzed using the two-sample F-test. Accuracy and repeatability data are presented in Table 1. 
Significant increases in accuracy were observed for the total rotation angle $\left(\theta_{\text {total }}\right)$ and dorsal angulation ( $\alpha$, about the lateral axis, $\mathrm{X}$ ) using the computer-assisted method $(p<0.01)$. Significant improvement in repeatability was observed in the computerassisted method for radial/ulnar rotation $(\beta$, about the anteroposterior axis, $\mathrm{Y}, p<0.1)$ and in radial/ulnar translations (along $\mathrm{X}, p<0.01$ ) and dorsal/volar translation (along $\mathrm{Y}, p<0.1$ ). There were no significant changes in accuracy of the other geometrical degrees of freedom, which were low in both groups.

Table 1. Accuracy and Repeatability comparisons. Errors are the difference between the planned and measured rotations and translations. The range is the difference between the largest and smallest error.

\begin{tabular}{|c|rrr|rrr|}
\hline & \multicolumn{3}{|c|}{ Errors, Traditional } & \multicolumn{3}{|c|}{$\begin{array}{c}\text { Errors, Computer-Assisted } \\
\end{array}$} \\
Mean Std. Dev. Range & Mean Std. Dev. & Range \\
\hline $\mathrm{x}(\mathrm{mm})$ & 1.9 & 3.8 & 10.7 & 0.4 & 1.2 & 3.0 \\
$\mathrm{y}(\mathrm{mm})$ & -1.7 & 3.7 & 11.64 & -1.0 & 2.1 & 5.3 \\
$\mathrm{z}(\mathrm{mm})$ & -0.1 & 1.8 & 6.0 & 0.8 & 1.7 & 4.6 \\
$\alpha(\mathrm{deg})$ & -10.3 & 3.0 & 9.2 & -1.1 & 2.9 & 7.1 \\
$\beta(\mathrm{deg})$ & -0.4 & 5.3 & 13.2 & 0.5 & 3.3 & 9.5 \\
$\gamma(\mathrm{deg})$ & 7.8 & 3.7 & 10.8 & 4.0 & 6.5 & 18.4 \\
$\theta_{\text {total }}(\mathrm{deg})$ & 14.0 & 4.0 & 10.9 & 7.7 & 3.5 & 11.12 \\
\hline
\end{tabular}

\section{A Pilot Clinical Study}

Four clinical procedures have been performed at Kingston General Hospital (Kingston, Ontario, Canada) to date. In the first procedure the correction achieved was as planned, but fluoroscopic validation suggested that the planned correction was short by approximately $2 \mathrm{~mm}$. This small additional correction was readily achieved intraoperatively. Subsequently, the planner was modified to provide contour rendering (which better displays concave features than does surface rendering). The next three cases all showed excellent alignment from fluoroscopic images and excellent postoperative results.

Case \#4 is a typical example of the use of our system. A 50-year-old male fell while traveling in Central America in February 1999. The immediate treatment, cast application without reduction, was unsuccessful. A severe deformity resulted (30 degrees of dorsal tilt, 15 degrees less of radial inclination, 8 degrees of supination, $12 \mathrm{~mm}$ of shortening), as shown in Figure 2. The consequence was pain, with functional impairment due to restricted motion and weakness.

The correction was planned as described above. The three views, shown in Figure 3, show the large amount of correction required to realign the deformity.

The final position and orientation of the articular radial surface was near anatomic, which is an excellent result. It is doubtful that this correction, shown in Figure 4 could have been achieved by conventional means. 


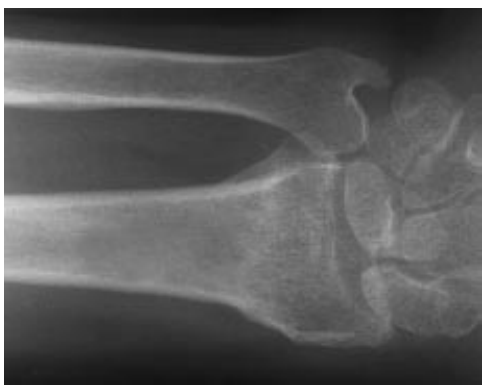

A

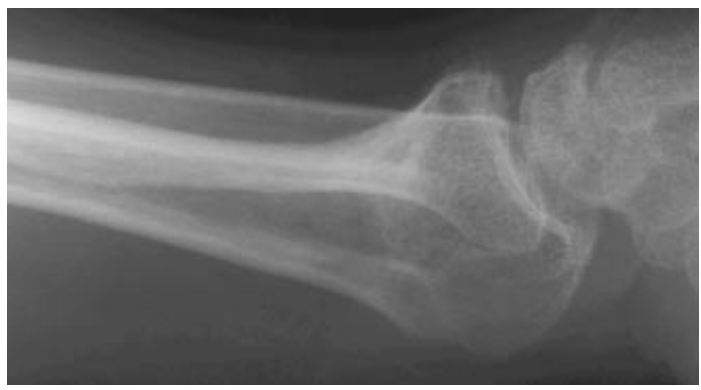

B

Fig. 2. Preoperative radiographs. (A) AP view of the malunited radius. Note the severe shortening and ulnar inclination (B) ML view of the malunited radius. Note the severe dorsal tilt.

\section{Discussion}

We report the design, implementation, and testing of a preoperative planning system that worked in conjunction with a guidance system to assist a surgeon in restoring the biomechanics and anatomy of the wrist. The ability of the system to simulate the osteotomy, and to view the expected results in solid and reduced renderings, allowed a surgeon to plan an osteotomy in a way that was far superior to the traditional film-based method. Laboratory results have shown that the system can improve the accuracy of producing a desired alignment.

Clinical trials have shown that the system is useful in improving surgical performance. An immediate benefit to the surgical team was that the amount of intraoperative radiation was reduced. Traditionally, numerous fluoroscopic images were required during the operation to determine the correct orientation of the distal radius after the cut. Using the image-based guidance system, X-ray images were needed only at the end of the procedure to verify the alignment.

Specific benefits of the planning system include the ability to perform multiple simulations of the surgical procedure preoperatively, which can be used to optimize the plan and identify potential problems during realignment (such as bone/bone contact). The planning system can also be used as a teaching tool to give medical students spatial visualization of deformities and ways of correcting them. Since the exact geometry of the created gap between the bones is known, CAD/CAM technology could be used to make artificial bone grafts, reducing the amount of trauma to the patient's body.

According to the literature reviewed, the three main anatomical measurements considered in correcting deformed distal radii are the ulnar variance, dorsal tilt, and radial tilt $[8,7,6,11]$. It was therefore not surprising to find that there was a large standard deviation in the other geometric variables, which were translation along the lateral and $\mathrm{A} / \mathrm{P}$ axes, and rotation about the pronation/supination axis. The results obtained using the computer-assisted corrections showed significant reductions in the other directions. 


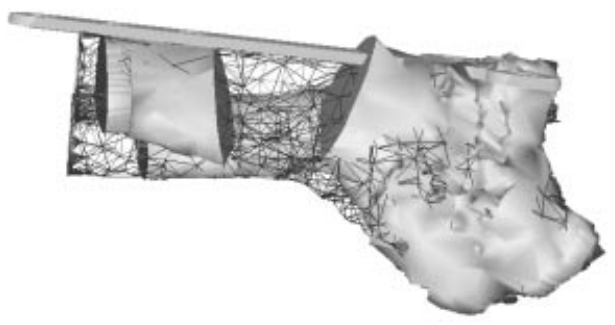

A

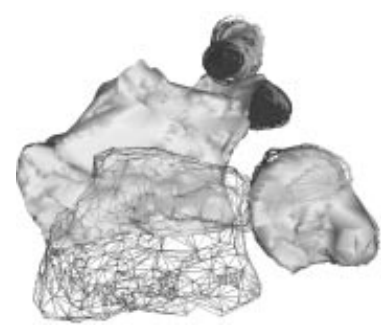

B

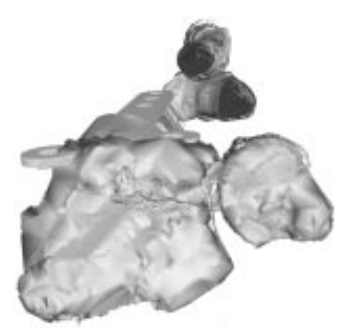

C

Fig. 3. Preoperative planning. (A) ML view of the planned correction, including the fixation plate. $(\mathrm{B}, \mathrm{C})$ Axial views of the original models and the planned correction.

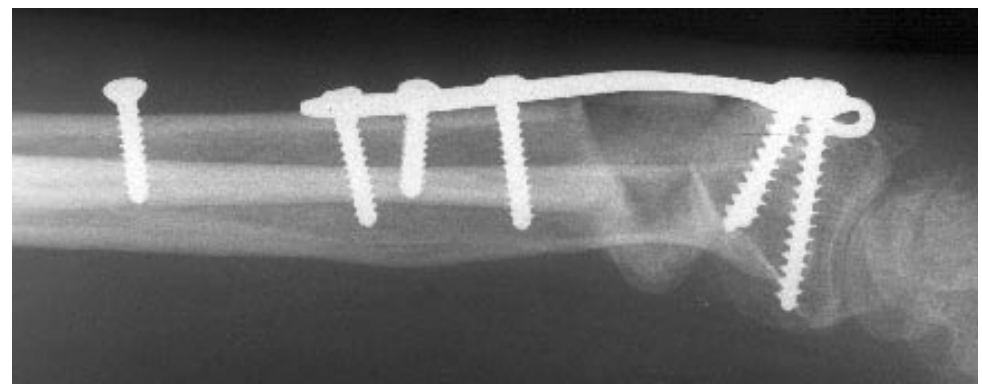

Fig. 4. Postoperative radiograph 8 weeks after surgery. The wrist has been restored to its anatomic alignment. Note the jacking screw. The bowing of the plate is due to the residual tension in the soft tissues.

Despite promising experimental and clinical results, some potential pitfalls are:

- The outcome of the surgery depends on the accuracy of the plan. Poor planning may result in poor surgical outcome.

- Due to the design of the fixation plate, the fixation screws are not always perpendicular to the surface of the plate. This must be checked in the planning process.

- When drilling the pilot holes, the drill could potentially bend. Bending is not accounted for by the tracking system.

- Placement of the plate depends on how well the images are registered to the patient's bone: a poor registration could result in poor surgical outcome. 
Fixation-based surgery is a method of planning and performing a surgical procedure in which the relative position of two bones is dictated by the fixation device that holds them together. For distal radius osteotomies, the holes for the screws in the fixation plate determined how the distal radius would lie relative to the proximal radius. The use of a fixation-based technique also avoids the complexity of attempting to guide the surgeon to realign a bone fragment in six degrees of freedom of correction.

Fixation-based surgery offers much to the practising hand surgeon. We are now examining ways in which the plate and fixation could be optimized for computer-assisted instumentation. The idea of fixation-based surgery might also be applicable in other surgical procedures.

\section{Acknowledgements}

This research was supported in part by Communications and Information Technology Ontario, the Institute for Robotics and Intelligent Systems, and the Natural Sciences and Engineering Research Council of Canada.

\section{References}

[1] R. Bilić and V. Zdravković. Planning corrective osteotomy of the distal end of the radius. 1. Improved Method. Unfallchirurg, 91:571-574, 1988.

[2] R. Bilić and V. Zdravković. Planning corrective osteotomy of the distal end of the radius. 2. Computer-aided planning and postoperative follow-up. Unfallchirurg, 91:575-580, 1988.

[3] R. Bilić, V. Zdravković, and Z. Boljević. Osteotomy for deformity of the radius. Computerassisted three-dimensional modeling. Journal of Bone \& Joint Surgery [Br], 76B(1):150 154, January 1994.

[4] A. J. Bronstein, T. E. Trumble, and A. F. Tencer. The Effects of Distal Radius Fracture Malalignment on Forearm Rotation: A Cadaveric Study. Journal of Hand Surgery [Am], 22A(2):258-262, March 1997.

[5] H. E. Cline, W. E. Lorensen, S. Ludke, C. R. Crawford, and B. C. Teeter. Two algorithms for the three-dimensional reconstruction of tomograms. Medical Physics, 15(3):320-327, May 1988.

[6] D. J. Pogue et. al. Effects of distal radius fracture malunion on wrist joint mechanics. Journal of Hand Surgery [Am], 15A(5):721-727, September 1990.

[7] J. Oskam et. al. Corrective osteotomy for malunion of the distal radius: The effects of concominant ulnar shortening osteotmy. Archives of Orthopaedic \& Trauma Surgery, 115(5):219-222, September 1996.

[8] D. L. Fernandez and J. B. Jupiter. Fractures of the Distal Radius: A Practical Approach to Management, chapter 2-5, 11. Springer Verlag, Inc., New York, 1996.

[9] J. B. Jupiter, J. Ruder, and D. A. Roth. Computer-generated bone models in the planning of osteotomy of multidirectional distal radius malunions. Journal of Hand Surgery [Am], 17A(3):406-415, May 1992.

[10] B. Ma, R. E. Ellis, and D. J. Fleet. Spotlights: A robust method for surface-based registration in orthopedic surgery. In Medical Image Computing and Computer-Assisted Intervention - MICCAI'99, pages 936-944. Springer Lecture Notes in Computer Science \#1496, 1999.

[11] M. Porter and I. Stockley. Fractures of the Distal Radius: Intermediate and End Results in Relation to Radiologic Parameters. Clinical Orthopedics, 220:241-251, July 1987.

[12] V. Zdravković and R. Bilić. Computer-assisted preoperative planning (CAPP) in orthopaedic surgery. Computer Methods \& Programs in Biomedicine, 32:141-146, 1990. 